\title{
Papers
}

\section{Determination of who may derive most benefit from aspirin in primary prevention: subgroup results from a randomised controlled trial}

\author{
T W Meade, P J Brennan on behalf of the MRC General Practice Research Framework
}

\begin{abstract}
Objective To determine which groups of patients may derive particular benefit or experience harm from the use of low dose aspirin for the primary prevention of coronary heart disease.

Design Randomised controlled trial.

Setting 108 group practices in the Medical Research Council's general practice research framework who were taking part in the thrombosis prevention trial. Participants 5499 men aged between 45 and 69 years at entry who were at increased risk of coronary heart disease.

Main outcome measures Myocardial infarction, coronary death, and stroke.

Results Aspirin reduced coronary events by $20 \%$. This benefit, mainly for non-fatal events, was significantly greater the lower the systolic blood pressure at entry (interaction $\mathrm{P}=0.0015$ ), the relative risk at pressures $130 \mathrm{~mm} \mathrm{Hg}$ being 0.55 compared with 0.94 at pressures $>145 \mathrm{~mm} \mathrm{Hg}$. Aspirin also reduced strokes at low but not high pressures, the relative risks being 0.41 and $1.42(\mathrm{P}=0.006)$ respectively. The relative risk of all major cardiovascular events - that is, the sum of coronary heart disease and stroke-was 0.59 at pressures $<130 \mathrm{~mm} \mathrm{Hg}$ compared with 1.08 at pressures $>145 \mathrm{~mm} \mathrm{Hg}(\mathrm{P}=0.0001)$.

Conclusion Even with the limitations of subgroup analyses the evidence suggests that the benefit of low dose aspirin in primary prevention may occur mainly in those with lower systolic blood pressures, although it is not clear even in these men that the benefit outweighs the potential hazards. Men with higher pressures may be exposed to the risks of bleeding while deriving no benefit through reductions in coronary heart disease and stroke.
\end{abstract}

\section{Introduction}

The use of aspirin for the primary prevention of coronary heart disease is already fairly widespread in many communities. As even small doses of aspirin in those who have not previously had clinical episodes may result in more harm than benefit ${ }^{1}$ and because (in primary prevention) large numbers must be treated to avoid one event, it is important to be as selective as possible in its use.

The primary prevention thrombosis prevention trial in men at increased risk of coronary heart disease recently showed that low intensity anticoagulation with warfarin to an international normalised ratio of about 1.5 and low dose aspirin $75 \mathrm{mg}$ daily each reduced the incidence of major episodes by about $20 \%$ over a median period of 6.8 years. ${ }^{2}$ In the case of warfarin, this reduction was mainly due to a decrease of $39 \%$ in fatal events, but it had little effect on non-fatal events. For aspirin, the reduction was due to a decrease of $32 \%$ in non-fatal events and a non-significant increase of $12 \%$ in fatal events. The design of the trial was factorial, so that the value of combined treatment-that is, with warfarin and aspirin-could be assessed. This reduced all coronary heart disease by $34 \%$, to which effects on both fatal and non-fatal events contributed.

The overall finding of the thrombosis prevention trial on aspirin was similar to that of the US physicians health study, in which the main result was a $44 \%$ reduction in non-fatal myocardial infarction. ${ }^{3}$ The results of the US trial also raised the possibility that aspirin may have been more effective in those aged 50 years or more and when cholesterol concentrations were low rather than high. These subgroup findings have been accorded some significance in guidelines on the use of aspirin in primary prevention. ${ }^{4}$ We therefore report similar findings from the thrombosis prevention trial to assess the consistency or otherwise of our findings with those of the US trial and to consider the implications for clinical practice.

\section{Methods}

The methods have been described in detail elsewhere, including the fulfilment of CONSORT criteria. ${ }^{256}$

Participants-The trial was carried out through 108 group practices in the Medical Research Council's general practice research framework in men aged between 45 and 69 years. At screening, history of smoking and family history of premature coronary heart disease were elicited, body mass index calculated, blood pressure measured, and blood samples taken for measurement of concentrations of total cholesterol and plasma fibrinogen and determination of plasma factor VII coagulant activity (VIIc). These variables

\author{
MRC Epidemiology \\ and Medical Care \\ Unit, Wolfson \\ Institute of \\ Preventive \\ Medicine, London, \\ EC1M 6BQ \\ T W Meade \\ director \\ P J Brennan \\ statistician \\ Correspondence to: \\ T Meade \\ t.w.meade@mds. \\ qmw.ac.uk
}

BMJ 2000;321:13-7 
were weighted according to their associations with coronary heart disease in the Northwick Park heart study, ${ }^{7}$ apart from family history which was not recorded in this study and which for the thrombosis prevention trial was considered to increase risk by $50 \%$. Within each practice, those men in the top $20 \%$ of the distribution of the risk score or in the top 25\% in regions with particularly high mortality from coronary heart disease were considered to be at increased risk and eligible for the trial. Of the 10557 men considered to be at high risk and eligible for the treatment phase, 5499 (52\%) entered the trial. Men came from all parts of the United Kingdom. Their mean (SD) age was 57.5 (6.7) years and just over $41 \%$ were current smokers. Their mean (SD) body mass index was 27.4 (3.6), and there was a family history of premature coronary heart disease in $15.5 \%$. Mean (SD) systolic blood pressure at entry was 139 (18) mm Hg. ${ }^{2}$ Antihypertensive drugs were being used by 245 men at the time of recruitment and were used by 1421 men at some stage during the trial. Mean systolic pressure during follow up was about $135 \mathrm{~mm} \mathrm{Hg}$.

Trial treatment-The factorial design of the trial, which was double blind and placebo controlled, resulted in four treatment groups-that is, active warfarin and active aspirin, active warfarin and placebo aspirin, placebo warfarin and active aspirin, and placebo warfarin and placebo aspirin. Warfarin was started at $2.5 \mathrm{mg}$ daily and adjusted by increments or decreases of $0.5 \mathrm{mg}$ or $1.0 \mathrm{mg}$ daily at monthly intervals until the international normalised ratio was about 1.5. Dose changes were matched in men on placebo warfarin. Aspirin was given as $75 \mathrm{mg}$ daily in a controlled release formulation. The main effect of aspirin was determined by comparing results in the combined warfarin and aspirin and aspirin only groups with those in the warfarin only and placebo groups and "main" is used only in this technical sense. (The main effects of warfarin, combined warfarin and aspirin, and warfarin only compared with aspirin only and placebo were not considered. See below).

Terminating events-The primary end point was all coronary heart disease, defined as the sum of fatal and non-fatal events (coronary death and all myocardial infarction). Stroke was a secondary end point.

Table 1 Coronary heart disease by age, systolic blood pressure, and cholesterol concentration according to treatment with aspirin. Figures are numbers of events/person years at entry with rates per 1000 person years in parentheses and relative risks corrected for other risk factors

\begin{tabular}{|c|c|c|c|c|}
\hline & Aspirin & No aspirin & Relative risk & $\begin{array}{l}\text { Interaction } \\
P \text { value }\end{array}$ \\
\hline \multicolumn{5}{|c|}{ Age (years): } \\
\hline $45-49$ & $14 / 3036(4.6)$ & $28 / 2930(9.6)$ & 0.48 & \multirow{5}{*}{0.055} \\
\hline $50-54$ & $26 / 3732(7.0)$ & $33 / 3831(8.6)$ & 0.77 & \\
\hline $55-59$ & $34 / 3410(10.0)$ & $37 / 3489(10.6)$ & 0.73 & \\
\hline $60-64$ & $36 / 3451(10.4)$ & $55 / 3325(16.5)$ & 0.61 & \\
\hline $65-69$ & $44 / 2601(16.9)$ & $37 / 2538(14.6)$ & 1.29 & \\
\hline \multicolumn{5}{|c|}{ Systolic blood pressure $(\mathrm{mm} \mathrm{Hg})$ : } \\
\hline$<130$ & $35 / 5475(6.4)$ & $56 / 5249(10.7)$ & 0.55 & \multirow{3}{*}{0.0015} \\
\hline $130-145$ & $40 / 5348(7.5)$ & $57 / 5338(10.7)$ & 0.75 & \\
\hline$>145$ & $79 / 5406(14.6)$ & $77 / 5526(13.9)$ & 0.94 & \\
\hline \multicolumn{5}{|c|}{ Cholesterol concentration (mmol/l): } \\
\hline$<5.9$ & $48 / 5510(8.7)$ & $53 / 5552(9.5)$ & 0.96 & \multirow{3}{*}{0.85} \\
\hline $5.9-6.7$ & $39 / 5146(7.6)$ & $63 / 4892(12.9)$ & 0.55 & \\
\hline$>6.7$ & $58 / 5315(10.9)$ & $70 / 5398(13.0)$ & 0.95 & \\
\hline
\end{tabular}

Follow up-The records of men in the trial were flagged in the NHS central register, thus ensuring automatic notification of death and the cause of death. Men were reviewed by their general practitioners each year, in addition to which the research nurse annually searched the notes of all those taking part for possible terminating events whether or not the man was still taking trial treatment. The independent assessment of end points was carried out according to World Health Organization (WHO) criteria $^{8}$ as previously described. ${ }^{2}$

Bleeding-Information about episodes of bleeding was obtained and classified as major, intermediate, or minor. ${ }^{2}$ Here, major and intermediate episodes are referred to as clinically significant.

Statistics-Rates are shown per 1000 person years together with relative risks. Interactions between the treatment effect of aspirin and all the variables used in the risk scoring were computed. As we had no prior hypotheses for most of the ensuing associations we restricted the principal findings to age and cholesterol concentration, for comparison with the US physicians health study, and to the only interaction terms significant at the $1 \%$ level or less, which were between aspirin treatment and blood pressure. (There were no interactions at less than the $1 \%$ level between the effect of warfarin treatment and any of the risk variables.) For tabular presentation we used arbitrary cut off values. The significance of interactions between the treatment effect of aspirin and the three risk factors, however, was assessed by using the full range of values for all variables. Findings for each variable under consideration were corrected for intercorrelations between age (except when age itself was under consideration) and all of the other seven risk factors used in the risk scoring procedure. All analyses were by intention to treat.

\section{Results}

The findings on aspirin treatment according to age, cholesterol concentration, and blood pressure are shown in table 1 . As expected, event rates within both the aspirin treated and untreated groups generally rose with increasing levels of these three risk factors. The relative risk of an event according to treatment in those with systolic pressures of $>145 \mathrm{~mm} \mathrm{Hg}$ was 0.94 , suggesting neither a beneficial or harmful effect. Those with pressures of $<130 \mathrm{~mm} \mathrm{Hg}$, however, experienced a risk reduction of $45 \%$ (relative risk 0.55 ) and the interaction term was significant $(\mathrm{P}=0.0015)$. Non-fatal events, to which the overall benefit of aspirin was confined, ${ }^{2}$ largely explain this effect. The finding on interaction with age, barely significant even at the 5\% level, was contrary to that in the US trial. (There was in fact an interaction of aspirin with age, suggesting increased coronary deaths at older ages, the relative risk at 65-69 years being 2.13, $\mathrm{P}=0.018$ for interaction.) There was no suggestion of an interaction with cholesterol concentration.

Table 2 shows the result for stroke (all types). The results for those on one or other of the warfarin regimens have been omitted to avoid exaggerating any apparent benefit due to aspirin that might be suggested by analysis of main effects. (This possibility arises because five of the 10 episodes of cerebral haemorrhage occurred in men with pressures $>145$ $\mathrm{mm} \mathrm{Hg}$ in the combined warfarin and aspirin 
treatment group, reflected in a relative risk of 1.74 and to which warfarin almost certainly also contributed.) There was a significant interaction between the effect of aspirin on its own with blood pressure, with a relative risk of 0.41 at $<130 \mathrm{~mm} \mathrm{Hg}$ compared with 1.42 at $>145 \mathrm{~mm} \mathrm{Hg}(\mathrm{P}=0.006)$. The possible reduction in benefit with increasing cholesterol concentration was of marginal significance.

Data on coronary heart disease and stroke were combined in an analysis of the main effect of aspirin to show the result for all major cardiovascular events (table 3). The increasing benefit with lower pressure was highly significant (interaction $\mathrm{P}=0.0004$ ). (Omission of all haemorrhagic strokes resulted in relative risks of $0.59,0.68$, and $1.08(\mathrm{P}=0.0001)$ in the three pressure groups respectively.)

Similar results were obtained with diastolic pressure. There were no interactions between the effects of any of the three separate active regimens or of all three treatments combined and any of the characteristics with the risk of clinically significant bleeding.

\section{Discussion}

\section{Subgroup analysis}

Subgroup findings must be interpreted with great caution as they will often have arisen purely by chance. We should not, however, overlook results that may truly indicate which patients may derive particular benefit or be harmed or that may provide a strong case for further trials, although as there is obviously a practical limit to carrying out all trials that might be suggested, this in itself indicates the need for careful, qualified consideration of subgroup results. We had no prior hypothesis that the value of aspirin might vary with blood pressure. One explanation might be the greater likelihood or extent of rupture of atheromatous lesions at higher blood pressures with overwhelming tissue factor release and thrombus formation that aspirin cannot influence. The level of significance for the association between blood pressure and coronary heart disease (table 1), however, suggests that it may not have been due to chance. The nature of the association was similar for stroke (table 2), which shares many of the risk factors and pathological features characteristic of coronary heart disease.

\section{American and British trials}

Men in the thrombosis prevention trial were over half of those eligible from a representative population based sample and were selected according to several characteristics that placed them at increased risk of coronary heart disease, whereas participants in the US trial were a small proportion of a selected group and at low risk. The numbers of major coronary end point events for analysis were similar in the two trials, 378 in the US trial and up to 410 in the thrombosis prevention trial, even though there were about four times as many participants in the US trial. The doses of aspirin were $75 \mathrm{mg}$ daily in the thrombosis prevention trial and $325 \mathrm{mg}$ on alternate days in the US trial. There were more older men in the US trial than in the thrombosis prevention trial, but in general both trials were based on men in the fifth, sixth, and seventh decades. The most obvious difference in entry characteristics was that $41 \%$ of men in the thrombosis prevention
Table 2 Stroke by age, systolic blood pressure, and cholesterol concentration according to treatment with aspirin. Figures are numbers of events/person years at entry in each stratum with rates per 1000 person years in parentheses and relative risks corrected for other risk factors

\begin{tabular}{|c|c|c|c|c|}
\hline & Aspirin (only) & Placebo & Relative risk & $\begin{array}{l}\text { Interaction } \\
\mathrm{P} \text { value }\end{array}$ \\
\hline \multicolumn{5}{|l|}{ Age (years): } \\
\hline $45-49$ & $0 / 1447(0.0)$ & $1 / 1502(0.7)$ & NA & \multirow{5}{*}{0.85} \\
\hline $50-54$ & $3 / 1905(1.6)$ & $0 / 1899(0.0)$ & NA & \\
\hline $55-59$ & $4 / 1663(2.4)$ & $11 / 1812(6.1)$ & 0.40 & \\
\hline $60-64$ & 7/1811 (3.9) & 9/1756 (5.1) & 0.99 & \\
\hline $65-69$ & $4 / 1279(3.1)$ & $5 / 1102(4.5)$ & 0.59 & \\
\hline \multicolumn{5}{|c|}{ Systolic blood pressure $(\mathrm{mm} \mathrm{Hg})$ : } \\
\hline$<130$ & $2 / 2688(0.7)$ & $6 / 2687(2.2)$ & 0.41 & \multirow{3}{*}{0.006} \\
\hline $130-145$ & $2 / 2663(0.8)$ & $10 / 2582(3.9)$ & 0.21 & \\
\hline$>145$ & $14 / 2754(5.1)$ & $10 / 2802(3.6)$ & 1.42 & \\
\hline \multicolumn{5}{|c|}{ Cholesterol concentration (mmol/l): } \\
\hline$<5.9$ & $3 / 2808(1.1)$ & $12 / 2837(4.2)$ & 0.20 & \multirow{3}{*}{0.036} \\
\hline $5.9-6.7$ & $7 / 2674(2.6)$ & $8 / 2345(3.4)$ & 0.90 & \\
\hline$>6.7$ & $8 / 2471(3.2)$ & $6 / 2748(2.2)$ & 1.53 & \\
\hline
\end{tabular}

NA: no events in one treatment group.

trial were current smokers compared with $11 \%$ in the US trial. Tests for interaction or trend, however, did not suggest differential benefits according to smoking habit in either trial. There was an unexplained contrast between the ratios of fatal to non-fatal episodes in the two trials-about one to two in the thrombosis prevention trial compared with one to nine in the US trial, the latter being at variance with most other studies, which have generally found ratios nearer to those in the thrombosis prevention trial. ${ }^{9}$ What age effect there may have been in the US trial was significant only at $\mathrm{P}=0.02$, and the effect for cholesterol concentration was also marginally significant at $\mathrm{P}=0.04$.

The trend for systolic blood pressure in the US trial was similar to ours. The relative risks from the lowest to the highest of the four blood pressure groupings used were $0.22,0.52,0.55$, and 0.65 , but the trend was not significant $(\mathrm{P}=0.48)$. Taken at face value, however, this finding suggests some benefit of aspirin at all levels of pressure. The possible harm due to aspirin in older men in the thrombosis prevention trial (table 1, relative risk 1.29 in those aged 65-69) may go some way to explaining the (non-significant) excess of fatal events in the aspirin treated group as a whole. ${ }^{2}$ There is some evidence that this excess of fatal episodes may not simply have been a chance observation in the thrombosis prevention trial, ${ }^{10}$ although other data suggest that previous aspirin use may attenuate the severity of acute myocardial infarction. ${ }^{11} 12$

Overview of the secondary prevention trials does not suggest that aspirin has major differential effects in distinct subgroups. ${ }^{13}$ Secondary prevention trials do not include the substantial proportion of those who die during or soon after their first major episode of coron-

Table 3 Major cardiovascular events (coronary heart disease and stroke) by systolic blood pressure at entry according to treatment with aspirin. Figures are numbers of events with rates per 1000 person years in parentheses and relative risks corrected for other risk factors (see table 1 for person years)

\begin{tabular}{|c|c|c|c|c|}
\hline $\begin{array}{l}\text { Systolic blood pressure } \\
(\mathrm{mm} \mathrm{Hg})\end{array}$ & Aspirin & No aspirin & Relative risk & Interaction $P$ value \\
\hline$<130$ & $42(7.7)$ & $64(12.2)$ & 0.59 & \multirow{3}{*}{0.0001} \\
\hline $130-145$ & $48(9.0)$ & $75(14.0)$ & 0.68 & \\
\hline$>145$ & $111(20.5)$ & $99(17.9)$ & 1.08 & \\
\hline
\end{tabular}


ary heart disease, however, and the absence in primary prevention of any obvious effect of aspirin on coronary or cardiovascular mortality ${ }^{2314}$ contrasts with its value in reducing mortality (as well as morbidity) in secondary prevention. Participants in primary and secondary prevention trials may therefore differ in ways that mean aspirin will not necessarily have the same effects in these two settings, and attention has been drawn elsewhere to the heterogeneity of responses to aspirin..$^{10}$

\section{Balance of benefits and hazards}

Even for a 40 year old man who has so far not had coronary heart disease but is at increased risk, the likelihood of an event within a year may be less than the anticipated excess risk of cerebral haemorrhage, assessed chiefly for aspirin doses of $160 \mathrm{mg}$ or more. ${ }^{1}$ This risk may perhaps be avoided with $75 \mathrm{mg}^{214}$ but is not the only consideration. In the thrombosis prevention trial, aspirin prevented two or three non-fatal myocardial infarctions as a result of treating 1000 men for a year (a low return that is characteristic of primary compared with secondary prevention).

Self medication with aspirin is widespread, even by many for whom there is no particular indication of risk. If our results are correct, men with pressures of about $145 \mathrm{~mm} \mathrm{Hg}$ or more will derive little if any cardioprotective benefit from aspirin. They will, however, be exposed to the risk of troublesome and occasionally serious non-cerebral bleeding, even with $75 \mathrm{mg}$ aspirin. Thus, a case-control study in five centres in the United Kingdom found a relative risk of peptic ulcer bleeding of 2.3 in regular users of $75 \mathrm{mg}$ aspirin compared with controls. ${ }^{15}$ There might be no case for recommending aspirin use for men with higher blood pressure, although the recent British Hypertension Society guidelines ${ }^{16}$ state that aspirin for primary prevention should be considered "for hypertensive patients aged 50 years or older who have satisfactory control of their blood pressure"-that is, $<150 / 90 \mathrm{~mm}$ $\mathrm{Hg}$-an upper systolic limit higher than our results indicate. If the overall benefit of aspirin is confined to those with lower pressures, it may be that four or five myocardial infarctions would be avoided by treating 1000 men for a year, but the risk of serious non-cerebral bleeding would also need to be taken into account.

Although suggestive on their own, our findings could be taken as firm evidence for decisions in clinical practice only if they were confirmed by results from other primary prevention trials. The hypertension optimal treatment trial ${ }^{14}$ did not comment on the effect, if any, of blood pressure at entry on response to aspirin but found a significant benefit due to aspirin when systolic pressures of about $142 \mathrm{~mm} \mathrm{Hg}$ had been achieved. While there is still some uncertainty about the level above which use of aspirin may be inadvisable, this trial, our own results, and the British Hypertension Society guidelines all draw attention to the importance of satisfactory control of blood pressure for those in whom the prophylactic use of aspirin is being considered.

Participating general practices: Abersychan, Abingdon, Alexandria, Backwell, Baildon, Balsall Common, Banbury, Barrow-inFurness, Bath, Beckenham, Belfast, Borough Green, Bracknell, Bromsgrove, Buckie, Bungay, Burnham Market, Burnham-on-
What is already known on this topic

The US physicians health study of aspirin in the primary prevention of coronary heart disease suggested marginally greater benefits in older than younger men and in those with low rather than high cholesterol concentrations

\section{What this study adds}

The UK thrombosis prevention trial in men has not confirmed the US trial results but has found a significantly greater beneficial effect of $75 \mathrm{mg}$ aspirin daily at low rather than high systolic blood pressures, not only for coronary heart disease but also for stroke

The evidence suggests that aspirin for the primary prevention of major cardiovascular disease may be more beneficial the lower the blood pressure, a possibility that must be balanced against the risk of bleeding

Men with higher blood pressure derive no cardioprotective benefit from aspirin but will risk possible serious bleeding

Crouch, Burton-upon-Trent, Cambridge, Carnforth, Castle Bromwich, Cherry Valley, Coleford, Cosham, Craven Park, Cullompton, Darlington, Didcot, Dingwall, Durham, Elstree, Evesham, Exmouth, Filey, Folkestone, Glasgow, Glyncorrwg, Grangemouth, Guisborough, Harrow, Herne Bay, High Wycombe, Hildenborough, Hornsey, Horwich, Hyde, Keadby, Kidderminster, Leamington Spa, Ledbury, Lichfield, Lisburn, Liskeard, Liverpool, Malvern, Mansfield, Melksham, Midsomer Norton, Newmarket, Newtown, Northampton, Northolt, Pembroke, Romsey, Scunthorpe, Sheffield, Shepperton, Shipley, Southampton, Stamford, Stanmore, Stevenage, Stockton-on-Tees, Stratford-upon-Avon, Sutton-in-Ashfield, Tiverton, Tonbridge, Torrington Park, Tutbury, Wakefield, Walsall, Whitehaven, Woodley, Worcester, Workington, Worthing.

The field work was coordinated by the framework nursing staff in the unit and the framework's regional trainers and was carried out by the research nurses and doctors in the participating practices. We gratefully acknowledge the help of many other doctors, nurses, and laboratory and administrative staff in the MRC Epidemiology and Medical Care Unit.

Contributors: TWM and PJB wrote the paper on behalf of the MRC's general practice research framework and are responsible for its contents. TWM was responsible for the initiation, design, and overall conduct of the trial. PJB was responsible for all the data processing and computing and the statistical analyses.

Funding: Medical Research Council, British Heart Foundation, DuPont Pharma, and Bayer Corporation. GlaxoWellcome and Boehringer-Ingelheim provided warfarin free of charge during the pilot stage. DuPont Pharma provided warfarin, and Bayer Corporation provided aspirin free of charge during the main trial.

Competing interests: DuPont Pharma and Bayer have given financial assistance with attendance at meetings and honoraria for speaking to Professor Meade and paid into a discretionary fund to supplement the unit's allocation.

1 Boissel J-P. Individualizing aspirin therapy for prevention of cardiovascular events. JAMA 1998;280:1949-50.

2 General Practice Research Framework Thrombosis Prevention Trial. Randomised trial of low-intensity oral anticoagulation with warfarin and low-dose aspirin in the primary prevention of ischaemic heart disease in men at increased risk. A report from the MRC's general practice research men at increased risk. A report from the
framework. Lancet 1998;351:233-41.

3 Steering Committee of the Physicians Study Research Group. Final report on the aspirin component of the ongoing physicians study. N Engl JMed 1989;321:129-35.

4 Cairns JA, Théroux P, Lewis DH Jr, Ezekowitz MD, Meade TW, Sutton GC. Antithrombotic agents in coronary artery disease. Chest 1998;114: 611-33S. 
5 Meade TW, Wilkes HC, Stirling Y, Brennan PJ, Kelleher C, Browne W. Randomized controlled trial of low dose warfarin in the primary prevention of ischaemic heart disease in men at high risk: design and pilot study. Euro Heart J 1988;9:836-43.

6 Meade TW, Roderick PJ, Brennan PJ, Wilkes HC, Kelleher CC Extra-cranial bleeding and other symptoms due to low dose aspirin and low intensity oral anticoagulation. Thromb Haemost 1992;68:1-6.

7 Meade TW, Mellows S, Brozovic M, Miller GJ, Chakrabarti RR, North WRS, et al. Haemostatic function and ischaemic heart disease: principal results of the Northwick Park heart study. Lancet 1986;2:533-7.

8 World Health Organization Regional Office for Europe. Myocardial infarction community registers. Copenhagen: WHO, 1976. (Public Health in Europe, No 5.)

9 Löwel H, Dobson A, Keil U, Herman B, Hobbs MST, Stewart A, et al. Coronary heart disease case fatality in four countries: a community study. Circulation 1993;88:2524-31

10 Alexander JH, Harrington RA, Tuttle RH, Berdan LG, Lincoff AM, Deckers JW, et al. Prior aspirin use predicts worse outcomes in patients with non-ST-elevation acute coronary syndromes. Am J Cardiol 1999;83: $1147-51$
11 Col NF, Yarzebski J, Gore JM, Alpert JS, Goldberg RJ. Does aspirin consumption affect the presentation or severity of acute myocardial infarction? Arch Intern Med 1995;155:1386-9.

12 Garcia-Dorado D, Theroux P, Tornos P, Sambola A, Oliveras J, Santos M, et al. Previous aspirin use may attenuate the severity of the manifestation of acute ischaemic syndromes. Circulation 1995;92:1743-8.

13 Antiplatelet Trialists' Collaboration. Collaborative overview of myocardial infarction and stroke by prolonged antiplatelet therapy in various categories of patients. BMJ 1994;308:81-106.

14 Hansson L, Zanchetti A, Carruthers SG, Dahlof B, Elmfeldt D, Julius S, et al. Effects of intensive blood-pressure lowering and low-dose aspirin in patients with hypertension: principal results of the hypertension optima treatment (HOT) randomised trial. Lancet 1998;351:1755-62.

15 Weil J, Colin-Jones D, Langmam, Lawson D, Logan R, Murphy M, et al. Prophylactic aspirin and risk of peptic ulcer bleeding. BMJ 1995;310: 827-30.

16 Ramsay LE, Williams B, Johnston GD, MacGregor GA, Poston L, et al. British Hypertension Society guidelines for hypertension management 1999: summary. BMJ 1999;319:630-5.

(Accepted 16 March 2000)

\title{
Risk of acquiring Creutzfeldt-Jakob disease from blood transfusions: systematic review of case-control studies
}

\author{
Kumanan Wilson, Catherine Code, Maura N Ricketts
}

\begin{abstract}
Objective To determine the strength of association between history of blood transfusion and development of Creutzfeldt-Jakob disease. Data sources English and non-English language articles published from January 1966 to January 1999 were retrieved using a keyword search of Medline and Embase. These were supplemented by handsearching key journals and searching bibliographies of reviews. Study selection Two independent reviewers selected the relevant abstracts and articles. Articles were chosen that reported the results of case-control studies trying to identify rates of prior blood transfusion in patients with Creutzfeldt-Jakob disease and in controls.
\end{abstract}

Data extraction Odds ratios and information on study quality were extracted from the selected articles by two independent reviewers.

Data synthesis Five studies containing data on 2479 patients were included. Three of the five studies used medical or neurological patients as controls, the other two used population controls. Odds ratios for developing Creutzfeldt-Jakob disease from blood transfusion ranged from 0.54 to 0.89 . Four of the five studies had confidence intervals that crossed 1.0. The combined odds ratio was $0.70(95 \%$ confidence interval 0.54 to 0.89 ).

Conclusions Case-control studies do not suggest a risk of developing Creutzfeldt-Jakob disease from blood transfusion. Rather, a trend seems to exist towards a lower frequency of previous blood transfusion in patients with Creutzfeldt-Jakob disease than in controls. However, it is important to be aware of these studies' methodological limitations-primarily the choice of control population and reliability of recall of transfusion status.

\section{Introduction}

The possibility of iatrogenic transmission of Creutzfeldt-Jakob disease via blood transfusion has recently attracted increased attention owing to the known transmissibility of hepatitis $\mathrm{C}$ and HIV via blood. ${ }^{1}$ Owing to the rarity of Creutzfeldt-Jakob disease and the potentially long latency period case-control studies are well suited to determine if an association exists between Creutzfeldt-Jakob disease and blood transfusion. We have conducted a systematic review of the evidence of blood transmission of sporadic Creutzfeldt-Jakob disease from case-control studies. Studies of variant Creutzfeldt-Jakob disease were not included in the review.

\section{Methods}

We conducted a search of English and non-English language articles in the Medline database from January 1966 to January 1999. We also searched the Embase database from 1988 to 1999 . We supplemented this search by handsearching key journals and searching bibliographies of reviews.

Two authors independently evaluated the abstracts and the retrieved articles and also extracted data. Articles selected for the systematic review had to meet two criteria. They had to have studied patients with Creutzfeldt-Jakob disease and controls, and they had to have determined the rate of blood transfusion in patients with Creutzfeldt-Jakob disease and controls. Odds ratios were calculated using the Meta-analyst program. $^{2}$

\section{Results}

Division of Genera Internal Medicine, University of Ottawa, Ottawa, Ontario, Canada Kumanan Wilson assistant professor

Department of Medicine, Ottawa Hospital-Civic Campus,

1053 Carling

Avenue, Ottawa,

Ontario K1Y 4E9

Catherine Code

resident in internal

medicine

Room L410,

Department of

Surveillance and

Response, World

Health

Organization,

Avenue Appia,

CH-1211, Geneva

27, Switzerland

Maura N Ricketts medical adviser on human and animal health

Correspondence to: K Wilson, Civic Parkdale Clinic 737 Parkdale Avenue, Suite 414, Ottawa, Ontario, Canada K1Y 1J8 kwilson@lri.ca

BMJ 2000;321:17-9 